\title{
Depression in young adults
}

\author{
Daniel J. Smith \& Douglas H. R. Blackwood
}

Abstract Depression, as a heterogeneous collection of disorders, is likely to include subgroups that are more genetic in origin. In common with other neuropsychiatric disorders such as schizophrenia, Alzheimer's disease and Huntington's disease, earlier age at onset in depression is associated with higher genetic loading and poorer long-term outcome. Adolescents and young adults with depression are also at high risk of developing a bipolar illness. This article reviews depressive illnesses that occur for the first time in adolescence and young adulthood. Case studies are used to discuss atypical presentations and the evolving concept of bipolar-spectrum disorders.

Affective illnesses may present at any age, but it is becoming clear that patients who suffer from recurrent and severe forms of mood disorder often experience their first episode of illness early in life. The clinical presentation of depression at this stage of life can be atypical and is often complicated by personality difficulties and substance misuse. A significant proportion of young people presenting with recurrent depression will go on to develop a bipolar disorder, with important implications for future pharmacological treatment choices.

\section{Epidemiology}

In the UK, suicide is now the most common cause of death in young men between the ages of 25 and 34. Epidemiological studies suggest that although factors such as poor schooling, poverty and unemployment are important, the strongest risk factors for suicide in this group are a history of mental illness, and a family history of suicide or mental illness (Agerbo et al, 2002). In a psychological autopsy study of completed suicide in young people aged between 15 and 24, Houston et al (2001) found that 19 out of 27 individuals (70\%) had suffered from a mental illness and that depression was the most common diagnosis, affecting 15 (56\%) of those studied. Eight individuals $(30 \%)$ had had a personality disorder and nine $(33 \%)$ had had a comorbid psychiatric disorder. It is notable that very few of these young people were receiving psychiatric care when they died. The Government's recently published National Suicide Prevention Strategy (Department of Health, 2002) acknowledges the importance of improved recognition and treatment of mood disorders in young adults, particularly within the young male population.

Although most epidemiological studies estimate that around $5 \%$ of the adult population suffer from depression, there are relatively few studies focusing on populations of adolescents and young adults. Although depressive symptoms appear to be common - a recent Finnish study of young adults identified a 1-month prevalence for major depression of $10 \%$ - only a small proportion of this group are likely to present to mental health services (AaltoSetala et al, 2001).

Adolescents with sub-diagnostic levels of depressive symptoms show higher rates of earlyadulthood depression, substance misuse and adverse psychological and social functioning (AaltoSetala et al, 2002). When symptom severity reaches the threshold for diagnosis, there is a likelihood that depression will continue into early adult life (Harrington et al, 1990; Lewinsohn et al, 1999).

The Maudsley long-term follow-up study of child and adolescent depression (Fombonne et al, 2001a), which followed 149 participants over 20 years, found that $62 \%$ experienced a recurrence of major depression. Similarly, rates of suicidal behaviour were high, with $44 \%$ attempting suicide at least once (Fombonne et al, 2001b). Levels of social dysfunction and service utilisation were much higher than in the general adult population (Knapp et al, 2002).

Daniel Smith is a research fellow in the Division of Psychiatry at the University of Edinburgh (Kennedy Tower, Royal Edinburgh Hospital, Morningside Park, Edinburgh, EH10 5HF, UK. Tel: +44 (0)131 537 6526; fax: +44 (0)131 537 6129; e-mail: daniel.smith@ed.ac.uk). He is currently involved in a neuropsychological and genetic study of mood disorders in young adults (funded by the Kate Hodgson Memorial Fellowship). Douglas Blackwood is Professor of Psychiatric Genetics at the University of Edinburgh and an honorary consultant psychiatrist to Lothian Primary Care NHS Trust. 


\section{The emergence of gender differences} during adolescence

That women are twice as likely as men to have depression is a consistent finding in psychiatric epidemiology and is not simply a consequence of females being more likely to report, recall or seek help for depressive symptoms. Before puberty, boys are slightly more likely than girls to be depressed, but between the ages of 11 and 13 this trend is reversed, with girls outnumbering boys by two to one. This predominance of females over males persists for the next 35 to 40 years. Changes in gonadal steroids are only part of the explanation for this gender gap. Hormonal changes in adolescence, combined with dramatic changes in social environment and relationships, stimulate the development of greater affiliative needs in females such as a preference for intimacy and emotional responsiveness. One result of this is that adolescent girls can be left more vulnerable to the effects of negative life events, especially ones that have interpersonal consequences (Cyranowski et al, 2000).

\section{Progression from early-onset unipolar depression to bipolar disorder}

Age at onset of depression and severity of depressive episodes are important factors in determining rates of ultimate progression to bipolar disorder. Prepubertal onset of depression is a strong marker for bipolar disorder, with some studies finding that at least one-third of depressed children will develop bipolar disorder in adult life (Geller et al, 2001). In a 7-year prospective study of 28 out-patient adolescents with depression, Rao et al (1995) detected a rate of bipolar outcome of almost $20 \%$.

Rates of switching polarity are higher for those with more severe episodes of depression. A 15-year follow-up of 74 young adults hospitalised for unipolar depression found that $27 \%$ subsequently developed hypomania, with an additional 19\% experiencing at least one episode of mania (Goldberg et al, 2001). The presence of psychotic symptoms during the index depressive episode was strongly predictive of bipolar disorder, with psychotic depression eventually becoming bipolar in eight out of ten patients. Unsurprisingly, patients with a positive family history of mania were also at higher risk of an outcome of bipolar disorder.

\section{Aetiology}

Depression in young adults occurs as a result of the dynamic interaction of a wide range of risk factors.

\section{The role of genetics}

Adoption and family studies have established that depression runs in families and that most of this familiality occurs as a result of genetic rather than environmental influences (Sullivan et al, 2000). Unipolar depression, as a heterogeneous disorder, is likely to include subgroups that represent more genetic forms of depressive illness. Recurrent, earlyonset depression, defined as two or more episodes before the age of 25, is associated with a strong family history of affective disorder and appears to follow a particularly malignant course, with frequent recurrence, poor response to treatment and high psychiatric and physical comorbidity (Zubenko et al, 2001). Although the heritability estimate of major depression across the life span is between $31 \%$ and $42 \%$, recurrent early-onset depression carries an estimated heritability of $70 \%$, a figure which is close to estimates for bipolar disorder.

A recent family study of recurrent, early-onset depression found that over one-third of first-degree relatives and one-fifth of extended relatives had a history of depression (Zubenko et al, 2001). Segregation analysis of these families was consisent with a single major locus being responsible for the expression of the disorder (Maher et al, 2002). Findings such as these have given added impetus to genetic studies of affective disorders.

\section{Neuroticism}

Neuroticism, defined as a general vulnerability to neurotic breakdown under stress, is a heritable personality trait (Tambs et al, 1991) and has been positively associated with depression (Duggan et al, 1995; Roberts \& Kendler, 1999). In a longitudinal study of 897 young adults followed between the ages of 18 and 21 years, Krueger et al (1996) found a strong association between high premorbid neuroticism and the subsequent development of a depressive illness.

However, the relationship between neuroticism and depression is complicated. Genes that predispose to mood disorders overlap with those implicated in neuroticism (Kendler et al, 1993) and individuals with high levels of neuroticism are more likely to experience depression after stressful life events than those with low levels (Farmer et al, 2002). Furthermore, evidence is emerging for a significant person-environment interaction whereby individuals with high neuroticism scores select themselves into high-risk environments and as a result become more likely to experience stressful life events (van Os et al, 2001).

For young adults with high levels of neuroticism, a vicious circle can be hypothesised in which they 
are more likely to place themselves in high-risk situations and, as a result of a high genetic loading for depression, are less able to withstand the adverse effects of stressful life events when they occur.

\section{Early adversity}

Childhood physical, emotional and sexual abuse are established as important risk factors for the development of a range of psychiatric disorders in adult life and are increasingly recognised as important in early-adulthood psychopathology. Traumatic experiences can interfere with normal emotional and psychological development, with the result that abused or neglected individuals often struggle to negotiate the maturational tasks of adolescence and early adulthood (Brown et al, 1999).

The observation that not all abused individuals develop significant psychopathological disorders in later life suggests that our susceptibility to stress is heavily dependent on our genetic make-up. This notion of genetic resilience in some individuals is supported by recent work on depression in adolescent girls, which confirms that genetic factors play an important role in determining their level of susceptibility to environmental stress (Silberg et al, 2001).

\section{Life events}

Although it is established that negative life events can precipitate depression, the association is a complex one and probably operates in both directions. People with depression are more likely to generate stressful events, and individuals with a higher genetic loading for affective disorder are more likely to experience depression after a stressful event than those with low genetic loading (Kendler $\mathcal{E}$ Karkowski-Shuman, 1997; Silberg et al, 2001).

In recurrent depressive disorder, the association between life events and depression is strongest for early episodes and becomes weaker as the number of episodes increases (Ghaziuddin et al, 1990). Recurrent depressive episodes tend to become more autonomous and are progressively less linked to environmental adversity, a phenomenon which has been called 'kindling' (Post, 1992). Kindling tends to be most marked in individuals at low genetic risk of depression; those at high genetic risk tend to exhibit 'prekindling'. Prekindled individuals appear to become depressed after only minimal environmental provocation (Kendler et al, 2001). One important implication of this is the possibility that young people with a strong family history of affective disorder are constitutionally vulnerable to the effects of even minor psychosocial stressors.

\section{Substance misuse}

Drug and alcohol use in adolescence are important risk factors for the development of affective disorders in early adulthood and are likely to complicate the long-term course of depression. In a 5-year longitudinal study of 155 adolescent females, Rao et al (2000) found that 19\% developed a substance use disorder and that substance use was a marker for the eventual occurrence of depression. Conversely, when Lewinsohn et al (2000) followed 274 formerly depressed adolescents to age 24, two-thirds had experienced another depressive episode and, from the remaining third who had not, $77 \%$ were found to have a substance misuse disorder. This suggests that an episode of depression in adolescence, or a diagnosis of substance misuse, represents an opportunity for early intervention to prevent recurrence of both disorders in later life.

\section{Cannabis}

Cannabis use in the UK has now reached such a level that a majority of young people use it recreationally (Smart \& Ogborne, 2000). Although the association between cannabis and psychotic illness is well recognised, much less attention has been paid to the association with affective disorders. Evidence is emerging for an important relationship between cannabis use and depression (Degenhart et al, 2001). Although cross-sectional surveys in young people confirm strong correlations between cannabis and depression (Rey et al, 2002), they tell us little about the causal mechanisms in operation. It may be that those who are premorbidly depressed are more likely to use cannabis as a form of selfmedication (Paton et al, 1977). Alternatively, higher use in depressed groups may be related to confounding factors such as social deprivation, early adjustment problems and poor academic achievement (Kandel et al, 1986).

Two recent longitudinal studies support the view that regular cannabis use at a young age is a precursor of depression in early adulthood. In the first, Paton et al (2002) followed 1601 students between the ages of 14 and 21 and found that daily use in adolescence was associated with a significant risk of anxiety and depression by early adulthood. This was particularly true for teenage girls - those who used cannabis on a daily basis were five times more likely to have depression than were non-users.

In the second study, the New York State Children in the Community Study, which followed 736 children between the ages of 14 and 27, Brook et al (2002) found that regular cannabis use was strongly predictive of depression in young adulthood. Interestingly, those who began to use cannabis in their early teens were at much higher risk than those 
who began in their early twenties, suggesting that a critical period exists during which the brain is acutely sensitive to the pathological effects of cannabis.

\section{Alcohol}

As in older adults, there is significant comorbidity between alcohol misuse and depression in young adults (Fergusson et al, 2002). That alcohol use at a young age leads to a higher risk of depression in young adulthood is supported by the findings of the Children in the Community Study mentioned above. Earlier alcohol use significantly predicted not only depression but also any substance use disorder and alcohol dependence by age 27 (Brook et al, 2002).

\section{Pathophysiology: the neurogenic theory of depression}

In recent years the monoamine theory of depression has given way to a molecular and cellular theory that suggests that antidepressants work by producing sustained activation of second messenger systems such as cyclic adenosine monophosphate (cAMP). This in turn leads to increases in brain levels of neurotrophic factors such as brain-derived neurotrophic factor (BDNF) that can reverse the detrimental effects of stress in brain areas such as the cerebral cortex and hippocampus (Reid \& Stewart, 2001). The neurogenic theory of depression posits a central role for dysregulation of the hypothalamic-pituitary-adrenal (HPA) axis and adrenal steroid-induced changes in hippocampal function in the pathophysiology of depression (Duman et al, 1997; Drevets, 2000). It is supported not only by new insights into how antidepressants work, but also by a growing body of evidence from basic science and clinical studies (for reviews see D'Sa \& Duman, 2002; Kempermann, 2002).

High ratios of cortisol to dehydroepiandrosterone (DHEA) are consistent findings in affective disorders and contribute to hippocampal atrophy. Excess cortisol impairs neurogenesis in the hippocampus, whereas DHEA may offer some protection (Young et al, 2002). Prospective studies of adolescents with no past history of depression have found that those who subsequently become depressed have higher ratios of cortisol to DHEA than controls (Goodyer et al, 2000). It is suggested that this can occur by two separate routes: genetic predisposition (a high familial loading for affective disorder); and early adverse experiences such as childhood sexual abuse.

Although magnetic resonance imaging studies have demonstrated reduced hippocampal volume in patients with major depression (Sheline, 2000), the debate continues over whether this is a reflection of a premorbid vulnerability or a consequence of repeated episodes of illness (Shatzberg, 2002). One recent study is of considerable interest in addressing this issue because it examines the function and volume of the hippocampus in a case-control study of young adults with depression (MacQueen et al, 2003). Twenty never-treated depressed patients in their first-episode of depression were compared with 17 patients with a history of multiple episodes of depression and with matched controls. Hippocampal function was assessed using neuropsychological tests of recollection memory and verbal memory, and hippocampal volume was measured by magnetic resonance imaging. Significantly, both first- and multiple-episode depressed groups had impaired hippocampal function as assessed by neuropsychological testing. However, only the group with multiple previous episodes had evidence of reduced hippocampal volume. This suggests that reduced hippocampal volume does not pre-date illness onset, but rather is a consequence of repeated episodes of illness. The finding of functional abnormalities of the hippocampus present at an early stage of illness is an important one and suggests that subtle structural abnormalities may be present that are as yet undetectable by imaging. This study also suggested that hippocampal volume loss occurred at the greatest rate in the earliest years after illness onset. Should these findings be reproduced elsewhere, they will represent strong arguments for aggressive early intervention in young people with recurrent or chronic depressive illnesses.

The finding of neurocognitive deficits in young adults even during the early stages of their illness has important implications for the way in which psychoeducational and cognitive-behavioural interventions are delivered. Information and therapy should be provided in a way that takes account of cognitive impairments in areas such as recollection memory and verbal learning.

\section{Classification}

\section{The unipolar/bipolar dichotomy}

Kraepelin, writing in the 1920s, had a broad view of manic-depressive illness that encompassed not only less-severe, attenuated forms but also much of the domain of major depressive disorders. Several important studies in the second half of the 20th century divided this unitary model of affective disorders into unipolar and bipolar disorders. In the 1950s, Leonard observed from his cohort of patients with recurrent depression that those who also had a history of mania tended to report more 


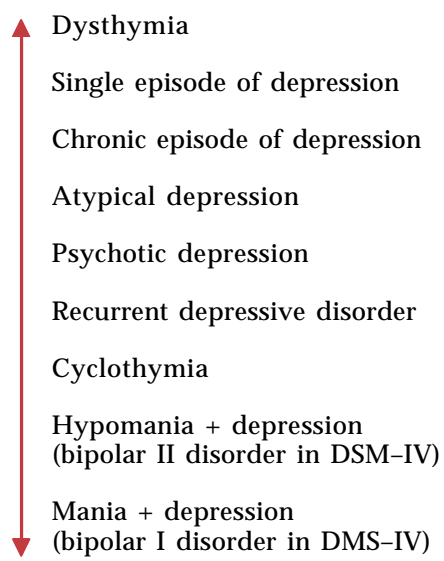

Fig. 1 The affective spectrum.

mania in their families than those who experienced only depressive episodes. In the 1960s, Angst and Perris provided independent family history data to support this. Several lines of evidence subsequently supported the concept of a unipolar/bipolar division: twice as many women as men experienced depression, whereas the ratio in bipolar disorder was 1:1; bipolar disorder tended to have an earlier age of onset; and mortality (mostly through suicide) was consistently higher in the bipolar group. This dichotomous conceptualisation is reflected in our contemporary classifications in ICD-10 (World Health Organization, 1992) and DSM-IV (American Psychiatric Association, 1994).

Although the distinction between unipolar and bipolar disorders has been a useful one, clinicians and researchers have long been aware that a significant proportion of patients with affective disorders do not fit comfortably into either of the two categories. Patients with mood disorders are increasingly seen as lying somewhere on a spectrum of severity ranging from dysthymia at one end to full-blown manic-depressive psychosis at the other (Fig. 1). In this model, classic 'manic depression' (bipolar I in DSM-IV and bipolar affective disorder in ICD-10) is a relatively infrequent presentation of the broad spectrum of affective illness.

The often-quoted prevalence rate of $1 \%$ for bipolar affective disorder is based on a DSM-III-R definition that requires at least 7 days of manic symptoms for the diagnosis of hypomania. We now know, however, that the modal duration of manic episodes in bipolar disorder is between 1 and 3 days, and recent epidemiological studies using less restrictive diagnostic criteria estimate that the true prevalence of bipolar disorders in the community is at least $5 \%$ (Angst, 1998). Furthermore, when patients with a diagnosis of depression are systematically assessed for a past history of manic symptoms, $45 \%$ satisfy diagnostic criteria for bipolar disorder (Allilaire et al, 2001). It is notable that levels of psychosocial impairment and service utilisation are similar between patients with broadly defined bipolar illness and those diagnosed using DSM-IV criteria (Judd \& Akiskal, 2003). Angst, who was one of the first to suggest that unipolar depression and bipolar disorder should be viewed as separate disorders, now argues that bipolar disorders have been underdiagnosed at the expense of depressive disorders and that between $25 \%$ and $50 \%$ of all patients with depression have in fact a bipolar disorder (Angst \& Gamma, 2002).

\section{Bipolar-spectrum disorder}

It is now 20 years since Akiskal and colleagues first demonstrated that certain clinical features of depressive episodes can predict bipolar outcome (Akiskal et al, 1983). These include depressive episodes of acute onset, frequent recurrence and brief duration; a family history of bipolar disorder; postpartum onset; retarded neurasthenic or atypical features; and pharmacological hypomania. Ghaemi et al (2002) have suggested that these features could form the basis of a diagnosis of bipolar-spectrum disorder for patients who do not meet the strict DSMIV criteria for bipolar disorder but who none the less have clinically significant bipolar features (Box 1).

Box 1 Proposed diagnostic criteria for bipolarspectrum disorder (reproduced with permission from Ghaemi et al, 2002)

A A major depressive episode

B No spontaneous DSM-IV hypomanic or manic episodes

C Either of the following plus two from D, or both plus one from $\mathrm{D}$ :

a First-degree relative with bipolar disorder

b Antidepressant-induced mania or hypomania

D If none from $C$, at least six of the following:

a Hyperthymic personality

b More than three depressive episodes

c Brief major depressive episodes (lasting less than 3 months)

d Atypical depressive symptoms

e Psychotic major depressive episodes

$f$ Early age of onset $(<25$ years old)

$g$ Post-partum depression

h Antidepressant 'wear-off' (acute but not prophylactic response)

i Lack of response to more than two antidepressant trials 


\section{Clinical presentation of depression}

Depression varies widely in its clinical presentation. This is especially true in adolescence and young adulthood, when atypical symptoms are more common and the more classic melancholic presentations are relatively rare (Parker et al, 2003). Younger adults with depression also tend to report more irritability and anxiety and, as detailed above, there should be a high index of suspicion for the possibility of an emerging bipolar disorder.

\section{Case 1 Anxious-hostile presentation}

A 21-year-old student was assessed on an orthopaedic ward 2 days after a violent suicide attempt. He had jumped from a height of $60 \mathrm{~m}$, sustaining a broken leg, multiple cuts, severe bruising and a minor head injury. He had taken the decision to kill himself after 2 weeks of escalating anxiety, irritability, disturbed sleep and agitation in the lead up to some important exams. He denied suffering from low mood, anhedonia, fatigue or appetite changes. He had strong obsessional and perfectionist traits in his personality and had become convinced that, despite studying extremely hard, he would fail his exams and that life was no longer worth living. He had taken the decision to kill himself only a few hours before the suicide attempt. Twelve months previously he had been admitted briefly to a psychiatric ward after an impulsive overdose of antidepressant medication. This previous episode of depression had also been characterised by symptoms of anxiety and irritability rather than more classic depressive features. After transfer from the orthopaedic ward he made a good recovery as an in-patient on a general psychiatric ward and eventually responded well to venlafaxine at a dose of $225 \mathrm{mg}$ per day.

Case 1 is an example of depression in a young man with prominent anxiety, irritability and impulsivity. Although this kind of presentation is well recognised in adolescent populations, it does not fit easily into our current adult ICD-10 and DSM-IV diagnostic classifications (Parker et al, 1999). Depressions characterised by irritability and hostility, particularly in young men, frequently go undiagnosed and are often associated with dangerous impulsive behaviour and potentially lethal acts of self-harm.

\section{Bipolar disorders in young adults presenting with depression}

Given that bipolar disorders tend to present initially in a depressive phase, that the majority of people with bipolar illness experience their first episode in adolescence and that rates of progression from adolescent depression to bipolar disorder are about 20\% (Rao et $a l, 1995)$, there is a strong case for careful consideration of bipolar illness in young adults presenting with recurrent or chronic depressive episodes.

\section{Case 2 Bipolar-spectrum disorder}

A 19-year-old undergraduate was referred by her general practioner because of a failure to respond adequately to two different classes of antidepressant. She had presented 12 months earlier with an episode of depression with prominent anxiety symptoms and a recurrence of self-cutting (at age 14 she had cut herself for several months around the time of her parents' separation). When treated with fluoxetine up to $40 \mathrm{mg}$ per day she had experienced an exacerbation of anxiety symptoms, and when switched to amitriptyline up to a dose of $200 \mathrm{mg}$ per day she reported considerable fluctuation in her mood and a worsening of her cutting behaviour. She also described several separate periods when her mood would be elevated for about 24 hours, during which time she had racing thoughts, pressure of speech, overactivity and social disinhibition. These episodes would be followed by an abrupt change in mood and longer periods of depression, anergia and social withdrawal. Of note in her family history was her mother's diagnosis of bipolar affective disorder and multiple second-degree relatives on her mother's side who suffered from depression and alcohol misuse. Her younger sister, aged 16, had also recently been treated for depression. The clinical impression was that antidepressant drugs so far had been unhelpful and were leading to a destabilisation of mood and a worsening of self-cutting behaviour. Amitriptyline was discontinued and she was prescribed lamotrigine up to a dose of $200 \mathrm{mg}$ per day. This led to a gradual resolution of her mood symptoms and self-harm over the next 6 weeks and she remained well at review several months later.

Case 2 is an example of depression in a young woman who has a first-degree relative with bipolar disorder. She had been difficult to treat and antidepressants led to fluctuations in her mood with recurrent, brief periods of hypomania occurring against a background of residual depressive symptoms. This kind of treatment-emergent (or pharmacological) hypomania and a history of a first-degree relative with bipolar disorder have both been included in the proposed diagnostic criteria for bipolar-spectrum disorder (Box 1). It is likely that a significant proportion of young adults presenting with recurrent depression will satisfy these diagnostic criteria.

\section{Case 3 Bipolar depression}

A 20-year-old woman was referred by her general practitioner because she described mood swings. She gave a 4-month history of feeling depressed and irritable, with excessive fatigue, overeating, weight gain and hypersomnia. She was also drinking heavily, on average over 70 units per week. She felt that she first had problems with her mood at the age of 12 , when she became overactive and disruptive at school, having previously been reserved and conscientious. She described several different periods in her life when her mood seemed to switch suddenly between episodes of depression and short-lived periods of 
euphoria, with overactivity and decreased need for sleep. These periods of elevated mood always lasted less than 2 days and tended to give way to longer periods of low mood and irritability. She denied ever having harmed herself, but had been hospitalised three times as a teenager because of excessive alcohol consumption. She had a strong family history of affective disorder (both parents and an elder brother had had depression). The impression was that she was currently experiencing an episode of depression against a background of significant mood instability and longstanding alcohol misuse. There was nothing in her history or presentation to suggest that she had a personality disorder. She was treated with sodium valproate up to a dose of $800 \mathrm{mg}$ per day and made a good symptomatic recovery within 4 weeks.

This is a case of depression characterised by excessive fatigue, hyperphagia, weight gain and hypersomnia. These symptoms are typical of the features of bipolar depression. Several other 'clues to bipolarity' have been recognised and are listed in Box 2 (Pies, 2002). Even though this patient does not meet the DSM-IV criteria for bipolar affective disorder (that is, periods of depression alternating with hypomania of at least 4 days' duration) she describes considerable mood instability. Diagnosing a bipolar-spectrum disorder in this case rather than unipolar depression has important implications for future pharmacological treatment choices, particularly because antidepressants can act as mood destabilisers in patients with a bipolar diathesis, by triggering mania, mixed affective states and rapid-cycling (Altshuler et al, 1995). The American Psychiatric Association treatment guidelines for bipolar depression are clear that the use of antidepressants should be kept to a minimum, with mood stabilisers such as lithium and lamotrigine representing the treatments of choice (American Psychiatric Association, 2002).

Box 2 Clues to possible bipolarity (adapted with permission from Ghaemi et al, 2002 \& Pies, 2002)

- Early age of onset (before age 25 years)

- Multiple episodes of depression, each usually of short duration (less than 3 months)

- Racing thoughts during depressive episodes

- Hyperphagia and weight gain

- Lethargy or psychomotor slowing

- Poor or short-lived response to antidepressants

- History of bipolar disorder in first-degree relative or multiple family members affected by unipolar depression

- Psychotic features

- Post-partum onset

- Manic symptoms induced by electroconvulsive therapy or antidepressants

- Hyperthymic personality traits at baseline

\section{The overlap with personality disorder}

There is a great deal of debate about whether the primary diagnosis in a substantial proportion of cases of borderline personality disorder is more usefully one of a primary mood disorder. In young adults, there is considerable overlap between the symptoms of mood disorder and cluster B personality disorder, particularly with regard to mood lability, impulsivity and self-harm. An 11-year follow-up of early-onset depression demonstrated that those who went on to develop bipolar disorder had many of the features of borderline personality disorder when first assessed (Akiskal et al, 1995). Given the damaging long-term implications of a diagnosis of personality disorder early in life, it seems good practice to exclude a primary mood disorder in young adults presenting with behaviours that might initially be suggestive of personality pathology. This kind of approach is supported by a recent international consensus that when patients satisfy the diagnostic criteria for both bipolar disorder and borderline personality disorder, a bipolar diagnosis is preferred (Akiskal et al, 2000).

\section{Conclusions}

Depression that has its onset in adolescence or young adulthood represents a severe form of affective disorder associated with a range of poor long-term outcomes. It often arises in families where multiple firstand second-degree relatives have a mood disorder and is frequently complicated by substance misuse.

Although we have highlighted the high rate of progression to bipolar disorder in this group, and ways in which subtle indicators of bipolarity can be used to guide treatment choices, further studies of the validity of the proposed diagnostic criteria for bipolarspectrum disorder are clearly required.

Genetic, neuroendocrine and brain-imaging studies are unravelling the complex interaction of constitutional and environmental risk factors in earlyonset depression, but there remains a need for longterm prospective studies of young people in the early stages of illness. These studies will have to allow the integration of findings from a diverse range of disciplines, from basic and clinical neurosciences to social science and epidemiology.

\section{References}

Aalto-Setala, T., Marttunen, M., Tuulio-Henriksson, A., et al (2001) One-month prevalence of depression and other DSMIV disorders among young adults. Psychological Medicine, 31, 791-801.

Aalto-Setala, T., Marttunen, M., Tuulio-Henriksson, A., et al (2002) Depressive symptoms in adolescence as predictors of early adulthood depressive disorders and maladjustment. American Journal of Psychiatry, 159, 1235-1237. 
Agerbo, E., Nordentoft, M. \& Mortensen, P. B. (2002) Familial, psychiatric and socioeconomic risk factors for suicide in young people: nested case-control study. BMJ, 325, 74-77.

Akiskal, H. S., Maser, J. D., Zeller, P., et al (1995) Switching from 'unipolar' to bipolar II: an 11-year prospective study of clinical and temperamental predictors in 559 patients. Archives of General Psychiatry, 52, 114-123.

Akiskal H. S., Walker, P., Puzantian, V. R., et al (1983) Bipolar outcome in the course of depressive illness: phenomenologic, familial, and pharmacologic predictors. Journal of Affective Disorders, 5, 115-128.

Akiskal, H. S., Bourgeois, M. L., Angst, J., et al (2000) Reevaluating the prevalence of and diagnostic composition within the broad clinical spectrum of bipolar disorders. Journal of Affective Disorders, 59, 5s-30s.

Allilaire, J., Hantouche, E. G., Sechter, D., et al (2001) Frequence et aspects cliniques du trouble bipolaire II dans une étude multicentrique française: EPIDEP (with English abstract). Encephale, XXVII, 149-158.

Altshuler, L. L., Post, R. M., Leverich, G. S., et al (1995) Antidepressant-induced mania and cycle acceleration: a controversy revisited. American Journal of Psychiatry, 152, $1130-1138$

American Psychiatric Association (1994) Diagnostic and Statistical Manual of Mental Disorders (DSM-IV) (4th edn). Washington, DC: APA.

American Psychiatric Association (2002) Practice guidelines for the treatment of patients with bipolar disorder (revision) American Journal of Psychiatry, 159 (suppl. 4), 1-50.

Angst, J. (1998) The emerging epidemiology of hypomania and bipolar II disorder. Journal of Affective Disorders, 50, 143151.

Angst, J. \& Gamma, A. (2002) Prevalence of bipolar disorders: traditional and novel approaches. Clinical Approaches in Bipolar Disorders, 1, 10-14.

Brook, D. W., Brook, J. S., Zhang, C., et al (2002) Drug use and the risk of major depressive disorder, alcohol dependance, and substance use disorders. Archives of General Psychiatry, 59, 1039-1044.

Brown, J., Cohen, P., Johnson, J. G, et al (1999) Childhood abuse and neglect: specificity of effects on adolescent and young adult depression and suicidality. Journal of the American Academy of Child \& Adolescent Psychiatry, 38, 1490-1496.

Cyranowski, J., Ellen, F., Young, E., et al (2000) Adolescent onset of the gender difference in lifetime rates of major depression: a theoretical model. Archives of General Psychiatry, 57, 21-27.

Degenhart, L., Hall, W. \& Lynskey, M.T. (2001) Alcohol, cannabis and tobacco use among Australians: a comparison of their associations with other drug use disorders, affective and anxiety disorders, and psychosis. Addiction, 96, 1603-1614.

Department of Health (2002) National Suicide Prevention Strategy for England. London: Department of Health. http:// www.doh.gov.uk/mentalhealth/suicideprevention.htm

Drevets, W. C. (2000) Neuroimaging studies of mood disorders. Biological Psychiatry, 48, 813-829.

D'Sa, C. \& Duman, R. S. (2002) Antidepressants and neuroplasticity. Bipolar Disorders, 4, 183-194.

Duggan, C., Sham, P., Lee, A., et al (1995) Neuroticism: a vulnerability marker for depression, evidence from a family study. Journal of Affective Disorders, 35, 139-143.

Duman, R. S., Heninger, G. \& Nestler, E. J (1997) A molecular and cellular theory of depression. Archives of General Psychiatry, 5, 597-606.

Farmer, A., Redman, K., Harris, T., et al (2002) Neuroticism, extraversion, life events and depression. British Journal of Psychiatry, 181, 118-122.

Fergusson, D. \& Woodward, L. J. (2002) Mental health, educational, and social role outcomes of adolescents with depression. Archives of General Psychiatry, 59, 225-231.

Fombonne, E., Wostear, G., Cooper, V., et al (2001a) The Maudsley long-term follow-up of child and adolescent depression. I: Psychiatric outcomes in adulthood. British Journal of Psychiatry, 179, 210-217.

Fombonne, E., Wostear, G., Cooper, V., et al (2001b) The Maudsley long-term follow up of child and adolescent depression. 2: Suicidality, criminality and social dysfunction in adulthood. British Journal of Psychiatry, 179, 218-223.

Geller, B., Zimerman, B., Williams, M., et al (2001) Bipolar disorder at prospective follow-up of adults who had prepubertal major depressive disorder. American Journal of Psychiatry, 158, 125-127.

Ghaemi, S. N., Ko, J. Y. \& Goodwin, F. K. (2002) Cade's disease and beyond: misdiagnosis, antidepressant use and a proposed definition for bipolar spectrum disorder. Canadian Journal of Psychiatry, 47, 125-134.

Ghaziuddin, M., Ghaziuddin, N. \& Stein, G. S. (1990) Life events and the recurrence of depression. Canadian Journal of Psychiatry, 35, 239-242.

Goldberg, J. F., Harrow, M. \& Whiteside, J. E. (2001) Risk for bipolar illness in patients initially hospitalised for unipolar depression. American Journal of Psychiatry, 158, 1265-1270.

Goodyer, I. M., Herbert, J., Tamplin, A., et al (2000) First-episode major depression in adolescents. Affective, cognitive and endocrine characteristics of risk status and predictors of onset. British Journal of Psychiatry, 176, 142-149.

Harrington, R., Fudge, H., Rutter, M., et al (1990) Adult outcomes of child and adolescent depression. 1: Psychiatric status. Archives of General Psychiatry, 47, 465-473.

Houston, K., Hawton, K. \& Shepperd, R. (2001) Suicide in young people aged 15-24: a psychological autopsy study. Journal of Affective Disorders, 63, 159-170.

Judd, L. J. \& Akiskal, H. G. (2003) The prevalence and disability of bipolar spectrum disorders in the US population: reanalysis of the ECA database taking into account subthreshold cases. Journal of Affective Disorders, 73, 123131.

Kandel, D. B., Davies, M., Karis, D., et al (1986) The consequences in young adulthood of adolescent drug involvement. An overview. Archives of General Psychiatry, 43, 746-754.

Kempermann, G. (2002) Regulation of adult hippocampal neurogenesis - implications for novel theories of major depression. Bipolar Disorders, 4, 17-33.

Kendler, K. \& Karkowski-Shuman, L. (1997) Stressful life events and genetic liability to major depression: genetic control of exposure to the environment. Psychological Medicine, 27, 539547

Kendler, K. S., Neale, M. C., Kessler, R. C., et al (1993) A longitudinal twin study of personality and major depression in women. Archives of General Psychiatry, 50, 853-862.

Kendler, K., Thornton, L. \& Gardner, C. (2001) American Journal of Psychiatry, 158, 582-586.

Knapp, M., McCrone, P., Fombonne, E., et al (2002) The Maudsley long-term follow-up of child and adolescent depression. 3. Impact of comorbid conduct disorder on service use and costs in adulthood. British Journal of Psychiatry, 180, 19-23.

Kraepelin, E. (1921) Manic-depressive insanity and paranoia. Edinburgh: E. and S. Livingstone.

Krueger, R. F., Caspi, A., Moffitt, T. E., et al (1996) Personality traits are differentially linked to mental disorders: a multitrait-multi-diagnosis study of an adolescent birth cohort. Journal of Abnormal Psychology, 105, 299-312.

Lewinsohn, P. M., Rohde, P., Klein, D. N., et al (1999) Natural course of adolescent major depressive disorder. I. Continuity into young adulthood. Journal of the American Academy of Child \& Adolescent Psychiatry, 38, 56-63.

Lewinsohn, P. M., Rhode, P., Seeley, J. R., et al (2000) Natural course of adolescent major depressive disorder in a community sample: predictors of recurrence in young adults. American Journal of Psychiatry, 157, 1584-1591.

MacQueen, G. M., Campbell, S., McEwen, B. S., et al (2003) Course of illness, hippocampal function, and hippocampal volume in major depression. Proceedings of the National Academy of Sciences, 100, 1387-1392.

Maher, B., Marazita, M. L., Zubenko, W. N., et al (2002) Genetic segregation analysis of recurrent, early-onset depression: evidence for single major locus transmission. American Journal of Medical Genetics, 114, 214-221.

Parker, G., Wilheim, K., Mitchell, P., et al (1999) The influence of anxiety as a risk to early onset major depression. Journal of Affective Disorders, 52, 11-17. 
Parker, G., Roy, K., Hadzi-Pavlovic, D., et al (2003) Distinguishing early and late onset non-melancholic unipolar depression. Journal of Affective Disorders, 74, 131-138.

Paton, G. C., Coffey, C., Carlin, J. B., et al (2002) Cannabis use and mental health in young people: cohort study. BMJ, 325 1195-1198.

Paton, S., Kessler, R. \& Kandel, D. (1977) Depressive mood and adolescent illicit drug use: a longitudinal analysis. Journal of General Psychology, 92, 267-287.

Pies, R. (2002) The 'softer' end of the bipolar spectrum. Journal of Psychiatric Practice, 8, 189-195.

Post, R. (1992) Transduction of psychosocial stress into the neurobiology of recurrent affective disorder. American Journal of Psychiatry, 149, 191-201.

Rao, U. M., Ryan, N. D., Birmaher, B., et al (1995) Unipolar depression in adolescents: clinical outcome in adulthood. Journal of the American Academy of Child E Adolescent Psychiatry, 34, 566-578.

Rao, U., Daley, S. E. \& Hammen, C. (2000) Relationship between depression and substance use disorders in adolescent women during the transition to adulthood. Journal of the American Academy of Child $\mathcal{E}$ Adolescent Psychiatry, 39, 215-222.

Reid, I. C. \& Stewart, C. A (2001) How antidepressants work: new perspectives on the pathophysiology of depressive disorder. British Journal of Psychiatry, 178, 299-303.

Rey, J. M., Sawyer, M. G., Raphael, B., et al (2002) Mental health of teenagers who use cannabis. Results of an Australian survey. British Journal of Psychiatry, 180, 216-221.

Roberts, S. B. \& Kendler, K. S. (1999) Neuroticism and selfesteem as indices of the vulnerability to major depression in women. Psychological Medicine, 29, 1101-1109.

Shatzberg, A. F. (2002) Brain imaging in affective disorders: more questions about causes versus effects. American Journal of Psychiatry, 159, 1807-1808.

Sheline, Y. I. (2000) 3D MRI studies of neuroanatomic changes in unipolar major depression. The role of stress and medical comorbidity. Biological Psychiatry, 48, 791-800.

Silberg, J., Rutter, M., Neale, M., et al (2001) Genetic moderation of environmental risk for depression and anxiety in adolescent girls. British Journal of Psychiatry, 179, 116-121.

Smart, A. G. \& Ogborne, A. C. (2000) Drug use and drinking among students in 36 countries. Addictive Behaviors, 25, 455460 .

Sullivan, P., Neale, M. C. \& Kendler, K. S. (2000) Genetic epidemiology of major depression: review and meta-analysis. American Journal of Psychiatry, 157, 1552-1562.

Tambs, K., Sundet, J. M., Eaves, L., et al (1991) Pedigree analysis of Eysenck Personality Questionnaire (EPQ) scores in monozygotic (MZ) twin families. Behavior Genetics, 21, 369382.

van Os, J., Park, S. B. G. \& Jones, P. B. (2001) Neuroticism, life events and mental health: evidence for person-environment correlation. British Journal of Psychiatry, 178 (suppl. 40), s72s77.

World Health Organization (1992) The ICD-10 Classification of Mental and Behavioural Disorders. Geneva: WHO.

Young, A. H., Gallagher, P. \& Porter, R. J. (2002) Elevation of the cortisol-dehydroepiandrosterone ratio in drug-free depressed patients. American Journal of Psychiatry, 159, 1237 1239 .

Zubenko, G., Zubenko, W. N., Spiker, D. G., et al (2001) Malignancy of recurrent, early-onset depression: a family study. American Journal of Medical Genetics, 105, 690-699.

\section{Multiple choice questions}

1 Depression in young adults:

a is more common in males than in females

b affects $25 \%$ of those between the ages of 20 and 24

c has a good prognosis when compared with depression in older adults d can present as personality disorder

e rarely develops into bipolar disorder.

2 In the aetiology of early-onset depression:

a most of the familiality is a consequence of genetic rather than environmental influences

b earlier age at onset is often associated with a strong family history of affective disorder

c obsessional personality traits are more common than neuroticism

$\mathrm{d}$ the impact of early traumatic experiences can be attenuated by genetic factors

e the association between life events and depression becomes stronger as the number of depressive episodes increases.

3 Regarding the pathophysiology of depression:

a high ratios of DHEA to cortisol protect against hippocampal atrophy

b cortisol abnormalities arise only as a result of early adverse experiences

c neurocognitive impairments are greater in depression than in bipolar disorder

$\mathrm{d}$ reduced size of the prefrontal subgenual cortex is a consistent finding in recurrent depression

e dysfunction of the hypothalamic-pituitary-adrenal axis is the primary neurological abnormality in affective disorder.

4 The phenomenology of early-adult depression:

a can be identical to that of depression in old age

$\mathrm{b}$ is often complicated by psychiatric comorbidity

c has important similarities with the presentation of depression in adolescence

$\mathrm{d}$ can be similar to depressions that occur in the course of a bipolar illness

e is rarely complicated by drug or alcohol misuse.

5 Bipolar-spectrum disorders:

a are a recognised diagnostic category in DSM-IV

b affect only a small minority of people with recurrent depressive disorders

c are not associated with significant social and occupational impairment

$\mathrm{d}$ are best treated with brief courses of antidepressants

e are more common in males than in females. 